\title{
Anti-EphA2 Antibody-directed Liposomal Docetaxel Prodrug MM-310
}

\author{
National Cancer Institute
}

\section{Source}

National Cancer Institute. Anti-EphA2 Antibody-directed Liposomal Docetaxel Prodrug

MM-310. NCI Thesaurus. Code C136981.

A formulation containing nanoparticles composed of liposomes that are conjug ated to ScFv antibody frag ments directed against the ephrin receptor A2 (EphA2; Ephrin A2) and a proprietary prodrug of docetaxel, a poorly water-soluble, second-generation taxane analog, with potential antineoplastic activity. Upon intravenous administration of the antiEphA2 antibody-directed liposomal docetaxel prodrug MM-310, the anti-EphA2 moiety selectively targets and binds to cells expressing Ephl3:112A2. Following accumulation of MM-310, docetaxel is slowly released from MM-310 and accumulates at the tumor site due to the unique characteristics of the tumor vasculature. In turn, docetaxel is taken up by tumor cells, where it binds to and stabilizes the beta-subunit of tubulin, thereby stabilizing microtubules and inhibiting microtubule disassembly. This results in cell cycle arrest and the induction of cell death. The cell-surface receptor EphA2, a member of the ephrin family of receptor tyrosine kinases (RT Ks) that are involved in mammalian development, is overexpressed by a variety of cancer cell types and plays an important role in tumor growth; its expression is associated with poor prognosis. Compared to free docetaxel, MM-310 increases docetaxel's half-life, and provides enhanced and specific accumulation in EphA2-expressing tumors, thereby increasing docetaxel's efficacy while lowering its systemic toxicity. 